\title{
POSSIBLE VELOCITY DISTURBANCES IN THE LAG ZONE AND ON THE FREE BOUNDARY IN A VISCOPLASTIC STRIP UNDER ROLLING
}

\author{
V. D. Solovei* \\ Institute of Engineering Science, Ural Branch of the Russian Academy of Sciences, 34 Komsomolskaya st., \\ Ekaterinburg, Russian Federation \\ *Corresponding author. E-mail: Solovei@yandex.ru ; address for correspondence: ul. Komsomolskaya, 34, 620049, \\ Ekaterinburg, Russian Federation. Tel.: +7 (343) 375-35-92; fax: +7 (343) 374-53-30
}

The isothermal slow plane flow of a viscoplastic strip under rolling is considered. The Prandtl friction law is assumed to be valid on the contact surface between the strip and the roller. Integral equations are found for possible tangential velocity disturbances in the lag zone and normal velocity disturbances on the free boundary for the special stationary flow of a strip at any time moment.

Keywords: slow flow of a viscoplastic strip under rolling, special stationary flow, possible disturbances of a special stationary flow, minimum entropy production.

DOI: $10.17804 / 2410-9908.2015 .4 .052-062$

\section{References}

1. Gorelik S.S. Rekristallizatsiya $v$ metallakh $i$ splavakh [Recrystallization in metals and alloys]. Moscow, Metallurgia Publ., 1978, 568 p. (In Russian).

2. Gubernatorov V.V., Sokolov B.K., Gervasyeva I.V., Vladimirov L.R. Formation of band structure in structurally homogeneous materials during deformation. Fizicheskaya mezomekhanika, 1999, vol. 2, no. 1-2, pp. 157-162. (In Russian).

3. Gubernatorov V.V., Sycheva T.S., Vladimirov L.R., Matveeva V.S., Pyatygin A.I., Melnikov M.B. Corrugation and formation of structure and texture in metallic materials during deformation and recryctallization: 2. Alloys of cubic system. Fizicheskaya mezomekhanika, 2002, vol. 5, no. 6, pp. 95-99. (In Russian).

4. Glensdorf P., Prigozhin I. Termodinamicheskaya teoriya struktury, ustoychivosti i fluktuatsiy [Thermodynamic theory of structure, stability and fluctuations]. Moscow, Mir Publ., 1973, 280 p. (In Russian).

5. Solovei V.D. Stability of flow and Prigogine's theorem for the stationary flow of a viscoplastic body. Inzhenerno-fizichesky zhurnal, 2011, vol. 84, no. 6, pp. 1293-1296. (In Russian).

6. Lamb G. Gidrodinamika [Hydrodynamics]. Moscow-Leningrad, GITTL Publ., 1947, 930 p. (In Russian).

7. Ilyushin A.A. Deformation of a viscoplastic body. Uchebniye zapiski MGU. Mekhanika, 1940, no. 39, pp. 3-81. (In Russian).

8. Freudental A., Geiringer H. Matematicheskie teorii neuprugoy sploshnoy sredy [The mathematical theories of the inelastic continuum]. Moscow, Fizmatgiz Publ., 1962, 432 p. (In Russian).

9. Kolmogorov V.L. Mekhanika obrabotki metallov davleniem [Mechanics of metal forming]. Moscow, Metallurgia Publ., 1986, 688 p. (In Russian).

10. Grudev A.P. Teoriya prokatki [Theory of rolling]. Moscow, Metallurgia Publ., 1988, 239 p. (In Russian).

11. Alekseev A.E. Nonlinear laws of dry friction in contact problems for the linear theory of elasticity. Prikladnaya mekhanika $i$ tekhnicheskaya fizika, 2002, vol. 43, no. 4, pp. 161-169. (In Russian). 
open-acesss journal

12. Ishlinsky A.Yu. On the stability of the viscoplastic flow of a strip and a round rod. Prikladnaya matematika i mekhanika, 1943, vol. VII, pp. 109-130. (In Russian).

13. Solovei V.D. Possible velocity disturbances in the lag zone in the rolling of a perfectly plastic strip. Deformatsiya i razrushenie materialov, 2014, no. 12, pp. 23-26. (in Russian).

14. Solovei V.D. Trukhin V.B. The stability of upsetting of a viscoplastic parallelepiped without friction. Deformatsiya i razrushenie materialov, 2014, no. 2, pp. 9-13. (in Russian). 
Подана в журнал: 19.06 .2015

УДК 559.374.2

DOI: $10.17804 / 2410-9908.2015 .4 .052-062$

\title{
ВОЗМОЖНЫЕ ВОЗМУЩЕНИЯ СКОРОСТЕЙ ПЕРЕМЕЩЕНИЙ В ЗОНЕ ОТСТАВАНИЯ И НА СВОБОДНОЙ ГРАНИЦЕ ВЯЗКОПЛАСТИЧЕСКОЙ ПОЛОСЫ ПРИ ПРОКАТКЕ
}

\author{
В. Д. Соловей*
}

Федеральное государственное бюджетное учреждение науки Институт машиноведения Уральского отделения Российской академии наук, ул. Комсомольская, 34, Екатеринбург, Российская Федераџия

*Ответственный автор. Электронная почта: SoloveiVD@yandex.ru ; адрес для переписки: ул. Комсомольская, 34, Екатеринбург, Российская Федерация. Телефон: +7 (343) 375-35-92; факс: +7 (343) 374-53-30

Рассматривается изотермическое медленное плоское течение вязкопластической полосы при прокатке. Трение на контакте полосы с валками определяется законом Прандтля. Найдены относящиеся к произвольному моменту времени интегральные уравнения для возможных возмущений касательных скоростей перемещений в зоне отставания на границе полосы с валком и нормальных скоростей перемещений на свободной границе полосы особого стационарного течения полосы.

Ключевые слова: медленное течение вязкопластической полосы при прокатке, особое стационарное течение, возможные возмущения особого стационарного течения, минимум производства энтропии.

\section{1. Введение}

Формирование периодических мезоструктур в металлах при прокатке [1] связано с потерей устойчивости деформируемых полос $[2,3]$. Исследование деформации твердого тела на устойчивость производится по отношению к некоторому множеству возмущений скоростей перемещений, удовлетворяющих дифференциальным уравнениям, вытекающим из законов сохранения, уравнениям состояния и граничным условиям [4]. При исследовании устойчивости особого стационарного течения вязкопластической полосы [5] при прокатке необходимо знать в произвольный момент времени возможные возмущения нормальных скоростей перемещений на свободной границе полосы и касательных скоростей перемещений в зоне отставания на контакте полосы с валком для особого стационарного течения.

В настоящей работе на основе закона сохранения мощности и принципа наименьшего производства энтропии Пригожина для произвольного момента времени выводятся граничные интегральные уравнения для возможных возмущений нормальных скоростей перемещений на свободной границе полосы и касательных скоростей перемещений в зоне отставания на контакте полосы с валком особого стационарного течения полосы.

\section{2. Основное особое стационарное течение вязкопластической полосы при прокатке}

Будем рассматривать плоское медленное течение вязкопластической полосы, для которого уравнения движения имеют следующий вид $[4,6]$ :

$$
\rho v_{i, t}=\sigma_{i j, j} .
$$

Здесь $v_{i}$ - компоненты вектора скорости перемещения частиц; $\sigma_{i j}-$ компоненты тензора напряжений; $\rho$ - плотность тела $(\rho=$ const); тензорные индексы принимают значения прямоугольных декартовых координат $x, y$; по дважды повторяющимся тензорным

Solovei V. D. / Possible velocity disturbances in the lag zone and on the free boundary in a viscoplastic strip under rolling. 
индексам $i, j, k, l$ происходит суммирование по всем возможным их значениям; запятая перед индексом обозначает частную производную по соответствующей координате или по времени.

Система уравнений для стационарного течения вязкопластического тела имеет вид [7-9]:

$$
\begin{gathered}
\sigma_{i j, j}=0 ; \\
v_{i, i}=0 ; \\
s_{i j}=s_{i j}^{\prime}+s_{i j}^{\prime \prime}=2 \mu \varepsilon_{i j}+2 K \varepsilon_{i j} / H ; \\
\varepsilon_{i j}=\left(v_{i, j}+v_{j, i}\right) / 2 ; \\
s_{i j}=\sigma_{i j}-\sigma \delta_{i j} ;
\end{gathered}
$$

где $\varepsilon_{i j}$ и $s_{i j}-$ компоненты тензора скоростей деформаций и девиатора напряжений соответственно; $s_{i j}^{\prime}$ и $s_{i j}^{\prime \prime}$ - компоненты вязкого и пластического девиатора напряжений соответственно; $\sigma=\sigma_{i i} / 3$ - гидростатическое давление; $H=\sqrt{2 \varepsilon_{i j} \varepsilon_{i j}}-$ интенсивность скоростей деформации сдвига; $K$ и $\mu$ - пластическая постоянная (предел текучести на сдвиг) и коэффициент вязкости материала соответственно $(K>0, \mu>0) ; \delta_{i j}-$ символ Кронекера.

Уравнения состояния (4) получены в предположении, что плотность диссипативной функции $f$, а также её вязкая и пластическая составляющие

$$
f=f^{\prime}+f^{\prime \prime}=\mu H^{2} / 2+K H
$$

являются вязкопластическим, вязким и пластическим потенциалами $[7,8]$ :

$$
\begin{gathered}
s_{i j}=\partial f / \partial \varepsilon_{i j} \\
s_{i j}^{\prime}=\partial f^{\prime} / \partial \varepsilon_{i j}, \quad s_{i j}^{\prime \prime}=\partial f^{\prime \prime} / \partial \varepsilon_{i j} .
\end{gathered}
$$

Напряжения на границе области течения полосы $p_{i}$ определяются формулой Коши

$$
p_{i}=\sigma_{i j} n_{j},
$$

где $n_{j}$-компоненты единичной внешней нормали к границе области течения полосы.

Схема процесса плоской прокатки полосы приведена на рисунке. Область $A B D E F(S)$ - область течения полосы. На границе контакта полосы с валком $D E\left(l_{s 2}\right)$ имеет место проскальзывание частиц полосы по поверхности валка (это характерно для низких полос [10]). В нейтральной точке $N$ касательные скорости перемещений частиц полосы и валка равны. Касательные составляющие скоростей перемещений частиц полосы $v_{t}$ в зоне отставания $D N\left(l_{s 2}^{\prime}\right)$ меньше, а в зоне опережения $N E\left(l_{s 2}^{\prime \prime}\right)$ - больше окружной скорости валка. Справа и слева от области течения изображены передний $\left(S_{2}\right)$ и задний $\left(S_{1}\right)$ жесткие концы полосы, движущиеся со скоростями $V_{2}$ и $V_{1}$ соответственно. Границы контакта области течения с жесткими концами полосы $S_{2}$ и $S_{1}$ обозначены как $E F\left(l_{v 2}\right)$ и $A B\left(l_{v 1}\right)$ 
Opten-aCCESS journal

соответственно. Граница $B D\left(l_{p}\right)$ свободна от нагрузки, а граница $A F\left(l_{s 1}\right)$ представляет плоскость симметрии процесса прокатки.

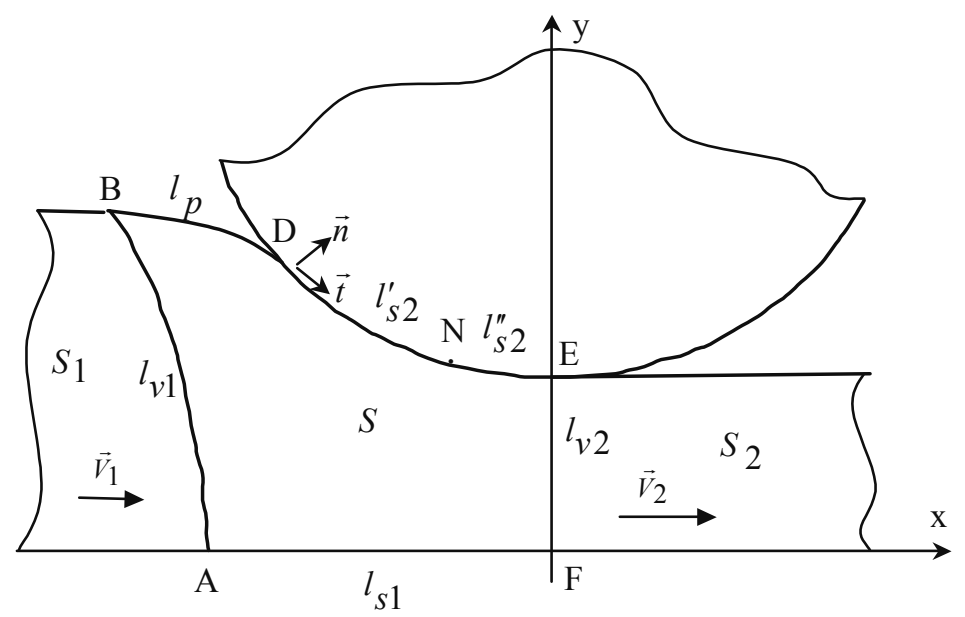

Схема прокатки полосы

Схема процесса плоской прокатки полосы приведена на рисунке. Область $A B D E F(S)$ - область течения полосы. На границе контакта полосы с валком $D E\left(l_{s 2}\right)$ имеет место проскальзывание частиц полосы по поверхности валка (это характерно для низких полос [10]). В нейтральной точке $N$ касательные скорости перемещений частиц полосы и валка равны. Касательные составляющие скоростей перемещений частиц полосы $v_{t}$ в зоне отставания $D N\left(l_{s 2}^{\prime}\right)$ меньше, а в зоне опережения $N E\left(l_{s 2}^{\prime \prime}\right)$ - больше, окружной скорости валка. Справа и слева от области течения изображены передний $\left(S_{2}\right)$ и задний $\left(S_{1}\right)$ жесткие концы полосы, движущиеся со скоростями $V_{2}$ и $V_{1}$ соответственно. Границы контакта области течения с жесткими концами полосы $S_{2}$ и $S_{1}$ обозначены как $E F\left(l_{v 2}\right)$ и $A B\left(l_{v 1}\right)$ соответственно. Граница $B D\left(l_{p}\right)$ свободна от нагрузки, а граница $A F\left(l_{s 1}\right)$ представляет плоскость симметрии процесса прокатки.

Граничные условия для основного течения имеют следующий вид:

$$
\begin{gathered}
p_{t}=p_{t}^{*}, \quad v_{n}=0 \text { на } l_{s 2} ; \\
p_{t}=p_{t}^{*}, \quad v_{n}=v_{n}^{*} \text { на } l_{v 1} ; \\
p_{t}=p_{t}^{*}, \quad v_{n}=V_{2} \text { на } l_{v 2} ; \\
p_{t}=0, \quad p_{n}=0 \text { на } l_{p} ; \\
p_{t}=0, \quad v_{n}=0 \text { на } l_{s 1} .
\end{gathered}
$$

Здесь $t$ и $n$-индексы компонент векторов в локальной системе координат $\vec{t}, \vec{n}$. Звездочка * обозначает, что соответствующая величина задана. 
Касательные напряжения трения $p_{t}$ на $l_{s 2}$ в (11) задаются приближенно с помощью закона трения Прандтля, который достаточно точно описывает закономерности внешнего трения в зонах больших нормальных напряжений [11]:

$$
p_{t}^{*}=K \text { на } l_{s 2}^{\prime}, \quad p_{t}^{*}=-K \text { на } l_{s 2}^{\prime \prime} \text {. }
$$

Эти напряжения трения в зоне отставания являются активными (их мощность положительна), а в зоне опережения-пассивными (их мощность отрицательна).

Касательные напряжения $p_{t}$ на границах $l_{v 1}$ и $l_{v 2}$ также задаются приближенно, для них:

$$
p_{t}^{*}=K
$$

Эти напряжения являются пассивными.

Выделим в тензоре напряжений вязкую и пластическую части:

$$
\sigma_{i j}=\sigma_{i j}^{\prime}+\sigma_{i j}^{\prime \prime}
$$

где

$$
\sigma_{i j}^{\prime}=s_{i j}^{\prime}+\sigma^{\prime} \delta_{i j}, \quad \sigma_{i j}^{\prime \prime}=s^{\prime \prime}{ }_{i j}+\sigma^{\prime \prime} \delta_{i j} .
$$

Здесь $\sigma_{i j}^{\prime}$ и $\sigma_{i j}^{\prime \prime}-$ вязкая и пластическая части тензора напряжений соответственно; $\sigma^{\prime}$ и $\sigma^{\prime \prime}$ - вязкая и пластическая части гидростатического давления. Величины, относящиеся к вязкой и пластической частям тензора напряжений, отмечаются одним ' и двумя "' штрихами соответственно. Так, напряжение на границе тела равно

$$
p_{i}=p_{i}^{\prime}+p_{i}^{\prime \prime},
$$

где $p_{i}^{\prime}$ и $p_{i}^{\prime \prime}-$ вязкая и пластическая части поверхностного напряжения соответственно.

Отметим, что граничные условия (11)-(13), учитывая (20), (10), (6) и (4), на границах $l_{s 2}, l_{v 1}$ и $l_{v 2}$ задают касательные напряжения в идеально пластическом приближении, т. е на этих границах вязкие части напряжений равны нулю:

$$
p_{t}^{\prime}=0 \text {. }
$$

Покажем также, что в принятом приближении пластические и вязкие касательные напряжения на границах $l_{p}$ и $l_{s 1}$ равны нулю. Для этого заметим, что, с одной стороны, используя соотношения (10), (18)-(20), (6) и (4) в локальных координатах, можно убедиться, что пластические и вязкие касательные напряжения на этих границах ( $p_{t}^{\prime \prime}$ и $\left.p_{t}^{\prime}\right)$ должны иметь одинаковый знак. С другой стороны, из тех же соотношений и первых выражений в (14) и (15) для рассматриваемых границ вытекают выражения

$$
p_{t}^{\prime}=-p_{t}^{\prime \prime} .
$$

Из сказанного следует, что на границах $l_{p}$ и $l_{s 1}$ пластические и вязкие касательные напряжения равны нулю:

$$
p_{t}^{\prime}=p_{t}^{\prime \prime}=0 \text {. }
$$

В работе [5] дано определение особого стационарного течения вязкопластического тела как течения, для которого вязкие и пластические напряжения порознь удовлетворяют уравнениям равновесия, т. е. 


$$
\begin{aligned}
& \sigma_{i j, j}^{\prime}=0 ; \\
& \sigma_{i j, j}^{\prime \prime}=0 .
\end{aligned}
$$

Из (23), (24), (18), (19) и (4) выводятся следующие соотношения, при выполнении которых стационарное течение вязкопластического тела является особым стационарным течением:

$$
2 K(1 / H) \varepsilon_{i j} H_{, j}+K \sigma_{, i}^{\prime} / \mu-H \sigma_{, i}^{\prime \prime}=0
$$

Из (25) видно, что стационарные течения с однородными скоростями деформаций, а также вязкими и пластическими частями гидростатического давления, являются особыми стационарными течениями. Такие особые стационарные течения имеют место, например, в процессах растяжения и сжатия вязкопластических полос [12].

Будем рассматривать только такие процессы прокатки полос, для которых особые стационарные течения, описываемые уравнениями (3)-(6), (23), (24), (18), (19) и граничными условиями (11)-(15), существуют. При этом в качестве основного течения вязкопластической полосы будем принимать особое стационарное течение.

Приведем выражение для закона сохранения мощности:

$$
W_{a}=W_{p} .
$$

Здесь мощность активных напряжений в зоне отставания $W_{a}$ и модуль мощности пассивных напряжений в объеме и на всех границах с трением (мощность диссипации) $W_{p}$ имеют вид:

$$
\begin{gathered}
W_{a}=\int_{l_{s 2}^{\prime}} p_{\mathrm{t}}^{*} v_{t} d l ; \\
W_{p}=\int_{S} w d S-\int_{l_{s 2}^{\prime \prime}} p_{\mathrm{t}}^{*} v_{t} d l-\int_{l_{v 1}} p_{t}^{*} v_{t} d l-\int_{l_{v 2}} p_{t}^{*} v_{t} d l ; \\
w=s_{i j} \varepsilon_{i j}=\mu H^{2}+K H=f+f^{\prime},
\end{gathered}
$$

где $w$ - плотность мощности диссипации.

Выражение (28) написано в предположении, что натяжения концов полосы отсутствуют [10], вследствие чего $p_{n}=0$ на $l_{v 1}$ и $l_{v 2}$.

Следует отметить, что для изотермического течения полосы производство энтропии $P$ связано с мощностью диссипации выражением [4]

$$
P=W_{p} / T,
$$

где $T$ - абсолютная температура.

Согласно теореме Пригожина, производство энтропии $P$ для особого стационарного течения вязкопластической полосы при прокатке минимально. Из (30) видно, что для изотермического особого стационарного течения вязкопластической полосы при прокатке мощность диссипации $W_{p}$ также должна быть минимальной. 
Opten-aCCESS journal

\section{3. Возможные возмущения скоростей перемещений основного течения в зоне отставания и на свободной границе вязкопластической полосы при прокатке}

Будем исследовать малые возмущения основного течения, которые описываются изохронными, изокоординатными вариациями переменных состояния $\delta v_{i}, \delta \varepsilon_{i j}, \delta \sigma_{i j}$, $\delta s_{i j}, \delta \sigma$, зависящими от координат и времени.

Система уравнений для возмущений основного течения вязкопластического тела имеет следующий вид:

$$
\begin{gathered}
\rho \delta v_{i, t}=\delta \sigma_{i j, j} ; \\
\delta v_{i, i}=0 \\
\delta s_{i j}=2 K H^{-3}\left(-2 \varepsilon_{k l} \delta \varepsilon_{k l} \varepsilon_{i j}+H^{2} \delta \varepsilon_{i j}\right)+4 \mu \delta \varepsilon_{i j} ; \\
\delta \varepsilon_{i j}=\left(\delta v_{i, j}+\delta v_{j, i}\right) / 2 ; \\
\delta s_{i j}=\delta \sigma_{i j}-\delta \sigma \delta_{i j} .
\end{gathered}
$$

Уравнения (31)-(35) получаются варьированием уравнений (1), (3)-(6) с удержанием только первых вариаций. При этом операция варьирования коммутирует с операциями взятия частных производных по координатам и времени.

Возмущения напряжений на границе области деформации $\delta p_{i}$ определяются формулой

$$
\delta p_{i}=\delta \sigma_{i j} n_{j} .
$$

Граничные условия для возмущений основного течения имеют следующий вид:

$$
\begin{aligned}
& \delta p_{t}=0, \quad \delta v_{n}=0 \text { на } l_{s 2} \\
& \delta p_{t}=0, \quad \delta v_{n}=0 \text { на } l_{v 1} \\
& \delta p_{t}=0, \quad \delta v_{n}=0 \text { на } l_{v 2} ; \\
& \delta p_{t}=0, \quad \delta p_{n}=0 \text { на } l_{p} ; \\
& \delta p_{t}=0, \quad \delta v_{n}=0 \text { на } l_{s 1} .
\end{aligned}
$$

Первая вариация мощности активных напряжений $\delta W_{a}$ согласно (27) имеет вид

$$
\delta W_{a}=\int_{l_{s 2}^{\prime}} p_{\mathrm{t}}^{*} \delta v_{t} d l
$$

Используя (29), (7), (8), (9), (6) и (32), симметрию тензора напряжений, а также теорему Гаусса-Остроградского, получим следующее соотношение:

$$
\delta \int_{S} w d S=-\int_{S}\left(\sigma_{i j, j}+\sigma_{i j, j}^{\prime}\right) \delta v_{\mathrm{i}} d S+\oint_{l}\left(p_{i}+p_{i}^{\prime}\right) \delta v_{i} d l
$$


Первую вариацию мощности диссипации $\delta W_{p}$ для особого стационарного течения, используя соотношения (28), (43), (23), (24) граничные условия (14), (21), а также первое выражение в (15) и вторые выражения в (37)-(39) и (41), представим в виде:

$$
\delta W_{p}=\int_{l_{s 2}^{\prime}} p_{t} \delta v_{t} d l+\int_{l_{p}}\left(p_{t}^{\prime} \delta v_{t}+p_{n}^{\prime} \delta v_{n}\right) d l+\int_{l_{s 1}} p_{t}^{\prime} \delta v_{t} d l
$$

Первая вариация мощности диссипации (44), учитывая (22), принимает вид:

$$
\delta W_{p}=\int_{l_{s 2}^{\prime}} p_{t} \delta v_{t} d l+\int_{l_{p}} p_{n}^{\prime} \delta v_{n} d l
$$

Варьируя выражение для закона сохранения мощности (26) и используя (42) и (45), получим первое интегральное уравнение для возможных возмущений нормальных скоростей перемещений на свободной границе полосы особого стационарного течения:

$$
\int_{l_{p}} p_{n}^{\prime} \delta v_{n} d l=0
$$

Нормальные напряжения $p_{\mathrm{n}}^{\prime}$ на свободной границе $l_{p}$ могут быть найдены из решения задачи основного течения вязкопластической полосы только для конкретных условий прокатки. Поэтому в общем случае уравнение (46) не может быть проинтегрировано. Отметим, что уравнение (46) существует только для вязкопластической полосы. Для идеально пластической полосы $(\mu=0)$ это уравнение вырождается.

Исходя из условия минимальной мощности диссипации $W_{p}$ в особом стационарном течении вязкопластической полосы при прокатке, сформулируем второе интегральное уравнение для возможных возмущений касательных скоростей перемещений в зоне отставания на границе контакта полосы с валком особого стационарного течения.

Рассмотрим сначала вторую вариацию мощности диссипации для произвольного течения вязкопластического тела $\delta^{2} W_{p}$. Учитывая (29), (7), (8) и (9), получаем

$$
\delta^{2} w=\left(\delta s_{i j}+\delta s_{i j}^{\prime}\right) \delta \varepsilon_{i j}
$$

Имеет место соотношение

$$
\delta s_{i j}^{\prime} \delta \varepsilon_{i j}=2 \mu \delta \varepsilon_{i j} \delta \varepsilon_{i j} \geq 0 .
$$

Докажем неравенство

$$
\delta s_{i j} \delta \varepsilon_{i j} \geq 0 \text {. }
$$

Левую часть (49), учитывая выражения (33), можно представить в виде

$$
\delta s_{i j} \delta \varepsilon_{i j}=4 K H^{-3}\left(\varepsilon_{i j} \varepsilon_{i j} \delta \varepsilon_{k l} \delta \varepsilon_{k l}-\varepsilon_{i j} \delta \varepsilon_{i j} \varepsilon_{k l} \delta \varepsilon_{k l}\right)+4 \mu \delta \varepsilon_{i j} \delta \varepsilon_{i j} .
$$

Поскольку выражение в скобках, согласно неравенству Коши-Буняковского, неотрицательно, то из (50) следует (49). диссипации

Из (28), (47), (49) и (48) следует, что достаточное условие минимальной мощности

$$
\delta^{2} W_{p} \geq 0
$$

удовлетворяется для произвольного течения вязкопластической полосы. к виду

Первую вариацию мощности диссипации (45), учитывая выражение (46), преобразуем

Solovei V. D. / Possible velocity disturbances in the lag zone and on the free boundary in a viscoplastic strip under rolling. 


$$
\delta W_{p}=\int_{l_{s 2}^{\prime}} p_{t} \delta v_{t} d l
$$

Принимая во внимание необходимое условие минимума мощности диссипации

$$
\delta W_{p}=0
$$

и соотношение (52), получаем второе интегральное уравнение для возможных возмущений касательных скоростей перемещений особого стационарного течения вязкопластической полосы в зоне отставания на границе контакта полосы с валком

$$
\int_{l_{s 2}^{\prime}} p_{t} \delta v_{t} d l=0
$$

Общее решение уравнения (54), принимая во внимание (16), можно представить в виде ряда на дискретном наборе волн возмущений [13]

$$
\delta v_{\mathrm{t}}=\sum_{k=1}^{\infty} a_{k} \sin \left[2 \pi k \frac{\left(l-l_{d}\right)}{\left(l_{n}-l_{d}\right)}\right],
$$

где $l$ - криволинейная координата на поверхности валка: $l=R \arcsin (x / R) ; l_{d}$ и $l_{n}-l-$ координаты, соответствующие декартовым координатам $x_{d}$ и $x_{n}$ точек $D$ и $N$ соответственно. Длина $k$-й волны возмущения равна $\lambda_{k}=\left(x_{n}-x_{d}\right) / k$. Коэффициенты разложения $a_{k}$ в (55) произвольны.

Отметим, что интегральное уравнение (54) формально имеет такой же вид, что и соответствующее уравнение для идеально пластической полосы [13]. Существенным является то, что в зоне отставания первое определяет возмущения $\delta v_{\text {t }}$ относительно особого стационарного течения, а второе - относительно стационарного течения.

Таким образом, возмущения скоростей перемещений в произвольный момент времени, используемые при исследовании устойчивости течения вязкопластической полосы при прокатке, должны удовлетворять не только уравнениям (31)-(35) и граничным условиям (37)-(41), но также и граничным интегральным уравнениям (46) и (54) или его решению (55). Устойчивость течения полосы по отношению к возмущениям скоростей перемещений в произвольный момент времени может быть исследована с помощью критерия локальной устойчивости в интегральном смысле, предложенного в работе [14].

\section{4. Заключение}

Найденные интегральные уравнения для возможных возмущений касательных скоростей перемещений в зоне отставания на границе контакта полосы с валком и нормальных скоростей перемещений на свободной границе полосы особого стационарного течения вязкопластической полосы при прокатке необходимо учитывать для построения возмущений скоростей перемещений в произвольный момент времени при исследовании устойчивости особого стационарного течения вязкопластической полосы при прокатке.

\section{Литература}

1. Горелик С. С. Рекристаллизация металлов и сплавов. - М. : Металлургия, 1978. $568 \mathrm{c}$.

2. Formation of band structures in homogeneous materials under deformation / V. V. Gubernatorov, B. K. Sokolov, I. V. Gervasyeva, L. R. Vladimirov // Physical Mesomechanics. - 1999. - Vol. 2, no. 1-2. - P. 157-162. 
3. The corrugation phenomenon and formation of strained and recrystallized structure and texture in metallic materials / V. V. Gubernatorov, L. R. Vladimirov, T. S. Sycheva, V. S. Matveeva, A. I. Pyatygin, M. B. Melnikov // Part II. Cubic syngony alloys. Physical Mesomechanics. - Vol. 5, no. 1-2. - P. 61-65.

4. Гленсдорф П., Пригожин И. Термодинамическая теория структуры, устойчивости и флуктуаций. - М. : Мир, 1973. - 280 с.

5. Solovei V.D. Flow stability and Prigogine theorem for the stationary flow of a viscoplastic body // Journal of Engineering Physics and Thermophysics. - 2011. - Vol. 84, no. 6. P. 1393-1397.

6. Ламб Г. Гидродинамика. - М.; Л. : ГИТТЛ, 1947. - 930 с.

7. Ильюшин А. А. Деформация вязко-пластичного тела // Уч. зап. МГУ. Механика. 1940. - Вып. 39. - С. 3-81.

8. Фрейденталь А., Гейрингер Х. Математические теории неупругой сплошной среды / пер. с англ. А. И. Смирнова; под ред. Э. И. Григолюка. - М. : Физматгиз, 1962. - 432 с.

9. Колмогоров В. Л. Механика обработки металлов давлением. - М. : Металлургия, 1986. $-688 \mathrm{c}$.

10. Грудев А. П. Теория прокатки. - М. : Металлургия, 1988. - 239 с.

11. Alekseev A. E. Nonlinear laws of dry friction in contact problems of linear theory of elasticity // Journal of Applied Mechanics and Technical Physics. - 2002. - Vol. 43, iss. 4. P. 622-629.

12. Ишлинский А. Ю. Об устойчивости вязкопластического течения полосы и круглого прута // Прикладная математика и механика. - 1943. - Т. VII. - С. 109-130.

13. Соловей В. Д. Возможные возмущения скоростей перемещений в зоне отставания при прокатке идеально пластической полосы // Деформация и разрушение материалов. - 2014. № 12 . - С. 23-26.

14. Соловей В. Д., Трухин В. Б. Устойчивость процесса осадки вязкопластического параллелепипеда без трения // Деформация и разрушение материалов. - 2014. - № 2. C. 9-13. 